\title{
A influência de fatores comportamentais e ambientais domésticos nas quedas em idosos
}

\section{The influence of behavior and domestic enviromental factors in elderly falls}

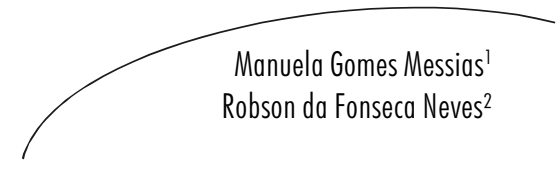

\section{Resumo}

O Brasil encontra-se entre os países da América Latina que têm experimentado maior aumento no número de pessoas da chamada terceira idade. No Brasil, aproximadamente $30 \%$ das pessoas com 65 anos e mais que moram em comunidades têm um evento de queda a cada ano. Metade dos idosos que caem repetem o evento. As lesões decorrentes dessas quedas são responsáveis pela sexta causa de morte nesta faixa etária. Tais episódios podem estar relacionados a fatores intrínsecos, bem como aos fatores extrínsecos e comportamentais. O presente estudo tem como objetivo revisar a literatura brasileira sobre fatores extrínsecos associados a quedas em idosos, descrevendo a participação de elementos físicos ambientais e comportamentais nesses eventos, além de sistematizar as principais recomendações sugeridas para prevenção das mesmas. Foram utilizados artigos publicados no período entre 1997 e 2007, acessados nas bases de dados eletrônicas; SciELO, Lilacs e Medline, os quais foram selecionados no idioma português utilizando-se os descritores: quedas, idosos. Concluiu-se, através da literatura consultada, que há conhecimento e soluções em abundância para eliminação dos fatores de risco físicos ambientais relacionados às quedas em idosos. Entretanto, os elementos comportamentais associados a estes eventos contemplados na literatura ainda são pouco estudados, o que pode dificultar tanto a elaboração, como o sucesso das estratégias de prevenção das quedas na população idosa.

\footnotetext{
Hospital de Base Luís Eduardo Magalhães, Itabuna, Bahia, Brasil.

2 Colegiado de Fisioterapia da Faculdade União Metropolitana de Educação e Cultura (UNIME), Lauro de Freitas, Bahia, Brasil
}

Correspondência / Correspondence

Manuela Gomes Messias

Av. Firmino Alves, 102/108 - Centro.

41600-000 - Itabuna, BA, Brasil

E-mail: manuelamessias@hotmail.com

Palavras-chave:

Acidentes por

Quedas. Idoso.

Ecologia Humana.

Fatores de Risco.

Prevenção de

Acidentes. Revisão.

Quedas. Riscos

Ambientais. Fatores

Comportamentais 


\section{Abstract}

Brazil is one Latin American countries that has experienced the largest increase in the number of elderly people. In Brazil, 30\% of the elderly who live in communities have one occurrence of fall every year. Half of them repeat the event. The injuries due to these falls are the sixth cause of death in this population. These episodes may be related with intrinsic factors, extrinsic factors and dangerous behavior. This study aims to review the Brazilian literature om extrinsic factors associated with elderly falls, describing the environmental elements and the elderly behavior in this events, and to systematize the main directions proposed to prevent falls. We examined published articles between 1997 and 2007 from the databases: SciELO, Lilacs, and Medline, which were selected in Portuguese by using the key words:

\section{Key words:}

Accidental Falls.

Aged. Human

Ecology. Risk Factors.

Accident Prevention.

Review. Falls.

Environmental

Hazard. Behavioral

Factors

falls, elderly. Based on the literature, we concluded that there are abundant knowledge and solutions for environmental risk factors related to elderly falls. However, the behavioral elements associated to these events present in literature are insufficiently assessed, what can hinder the elaboration and the success of preventive strategies of falls among the elderly population.

\section{INTRODUÇÃO}

No Brasil aproximadamente $30 \%$ das pessoas com 65 anos e mais que moram em comunidades, ou seja, que não foram institucionalizadas, têm um evento de queda a cada ano. Metade dos idosos que caem repetem o evento. As lesões decorrentes dessas quedas são responsáveis pela sexta causa de morte nessa faixa etária. Cerca de 5\% das quedas resultam em lesões graves e ocasionam mais de $200 \mathrm{mil}$ hospitalizações por fratura de quadril a cada ano. ${ }^{1,2}$ Tais episódios podem estar relacionados a processos intrínsecos ao envelhecimento, como por exemplo, a redução da acuidade visual e diminuição de força muscular, ou mesmo a fatores extrínsecos ao utilizarem mobiliário doméstico inadequado e mal posicionado, ou mesmo ao adotarem hábitos perigosos como subir em escadas móveis para alcançar armários altos.

O sistema de controle postural se deteriora durante o processo de envelhecimento. Isoladamente, essas alterações degenerativas podem parecer pequenas e insignificantes, mas a soma de déficits visuais, alterações osteomioarticulares e desorganização dos processos centrais aumentam o risco de ocorrer uma resposta incorreta ou insuficiente, resultando em perda da coordenação, sobretudo quando se trata de atividades funcionais complexas, tais como descer escadas carregando um objeto pesado ou deslocar-se rapidamente para o lado, a fim de evitar uma colisão.

De forma simplificada, um episódio de queda é resultado de uma interação de fato- 
res intrínsecos, decorrentes das alterações fisiológicas do próprio processo de envelhecimento e das patologias múltiplas associadas, e de fatores extrínsecos, ligados aos riscos ambientais somado aos fatores comportamentais, relacionados com o uso e percepção do espaço por parte do idoso frente à demanda imposta pelo ambiente e sua capacidade funcional real. As quedas não são consequência comum e inevitável da idade, mas uma séria ameaça para a saúde e a independência dos idosos. ${ }^{2}$

Pesquisas realizadas sobre o tema trazem informações acerca dos principais fatores relacionados a quedas em idosos, ${ }^{3-6}$ algumas vezes evidenciando os fatores ambientais, outras enfatizando os fatores intrínsecos. Também constataram os locais que mais predispõem às quedas, ${ }^{7-9}$ além de elaborarem propostas de projetos ambientais adequados e adaptados para idosos. ${ }^{5,10-12}$ Entretanto, ainda existe uma lacuna no conhecimento de como os fatores comportamentais podem contribuir com a ocorrência de quedas em idosos, sendo necessário esclarecer quais fatores estão sendo pouco abordados na literatura e quais recomendações podem ser estabelecidas como contribuição para prevenção de quedas em idosos na comunidade, para que se possa atuar através da estruturação de programas educativos, prática regular de exercícios, mudanças ou adapta- ções ambientais, assim como orientações para reduzir a probabilidade das quedas em idosos.

O presente estudo tem como objetivo revisar a literatura brasileira sobre fatores extrínsecos associados a quedas em idosos, descrevendo a participação de elementos físicos ambientais e comportamentais nestes eventos.

\section{MÉTODOS}

Trata-se de uma revisão da literatura sobre a influência dos fatores extrínsecos associados a quedas em idosos. Foram utilizados apenas artigos publicados no período entre 1997 e 2007. As publicações foram acessadas em bases de dados eletrônicas; SciELO* ${ }^{*}$ Lilacs $^{* *}$ e Medline $e^{* * *}$, as quais foram selecionadas no idioma português utilizando os seguintes descritores: quedas, idosos.

Como critérios de inclusão, foram utilizados artigos cuja temática abordasse os fatores físicos ambientais domésticos e comportamentais e as quedas em idosos. Foram excluídos todos os textos que se referiam apenas a idosos institucionalizados, e aqueles que discutiam exclusivamente fatores intrínsecos. Também foram excluídos os estudos que não se enquadraram no período

\footnotetext{
Scielo Express. Biblioteca Virtual de Saúde. Disponível em: http://www.scielo.org/php/index.php?lang=pt

** Lilacs Express. Biblioteca Virtual de Saúde. Disponível em: http://bases.bireme.br/cgi-bin/wxislind.exe/iah/online/ ? IsisScript $=$ iah $/$ iah.xis\&base $=$ LILACS\&lang $=p$

*** Medline Express. Biblioteca Virtual de Saúde. Disponível em: http://bases.bireme.br/cgi-bin/wxislind.exe/iah/ online $/$ ? IsisScript $=i a h /$ iah $. x i s \& b a s e=$ MEDLINE\&lang $=p$
} 
ou no idioma determinado para inclusão e que não utilizaram dados empíricos.

$\mathrm{Na}$ pesquisa, ao acessar a base de dados Lilacs utilizando os descritores selecionados, foram encontradas 74 publicações, dentre as quais apenas 12 se enquadraram nos critérios de inclusão. $\mathrm{Na}$ base de dados Medline foram encontradas 425 publicações, das quais apenas quatro atenderam aos interesses do estudo. E por fim, na base de dados SciELO foram encontrados 26 publicações, sendo utilizadas apenas sete, pelos mesmos motivos. Dos estudos selecionados, sete foram retrospectivos, cinco transversais, um prospectivo, um estudo de caso e um foi uma sugestão de projeto de habitação.

Os dados foram analisados comparandose os resultados obtidos pelos diversos autores em seus estudos, buscando similaridades e possíveis divergências entre si no que tange à relação entre fatores ambientais domésticos e comportamentais na ocorrência de quedas em idosos, visando a apontar os fatores extrínsecos relacionados a quedas mais evidenciados e os fatores pouco abordados na literatura atual. Visou-se, ainda, a sistematizar as principais recomendações sugeridas na literatura no que se refere à prevenção destes episódios.

\section{DISCUSSÃO}

A literatura brasileira ressalta a participação de fatores extrínsecos na ocorrência das quedas em idosos, porém ainda são escassas as publicações que tratam dos aspec- tos comportamentais relacionados a tais episódios. E como estratégias de prevenção, muitas recomendações têm sido apontadas no que se refere a segurança, programas educativos e treinamento de recursos humanos especializados na temática do idoso.

Existem muitos obstáculos ambientais que podem predispor o idoso a cair. $\mathrm{Na}$ comunidade, a maioria das quedas ocorre no próprio local de moradia, em lugares importantes, como escadas, quartos e salas. Os estudos brasileiros apresentam variações quanto ao setor da residência onde as quedas são mais frequentes: um estudo retrospectivo realizado com 56 idosos que sofreram fratura de quadril secundária à queda constatou como principais locais de ocorrência de quedas: em primeiro lugar, a cozinha e as escadas, seguidas pela sala, banheiro, quarto e quintal, e por último, o corredor. ${ }^{8}$ Ampliando este quadro, outro estudo com 64 idosos vestibulopatas em São Paulo verificou que no ambiente interno do domicílio o espaço mais apontado foi o banheiro, referido em 38,1\% das quedas relatadas. ${ }^{7}$ Por sua vez, foram observados em outra pesquisa os seguintes resultados: quarto $36,36 \%$; quintal $22,73 \%$; sala $13,64 \%$; banheiro $9,08 \%$; cozinha $4,55 \%$.

As principais causas extrínsecas de quedas estão relacionadas a aspectos físicos ambientais. Num estudo de prevalência realizado em São Paulo, observou-se que os riscos ambientais mais encontrados nos domicílios dos idosos participantes foram: piso escorregadio $65,5 \%$; presença de tapetes 
$62,1 \%$; presença de objetos desordenados 62,1\%; e armários difíceis de alcançar $51,7 \%{ }^{7}$ Outros fatores também estão relacionados à insegurança do idoso nos domićlios: iluminação inadequada, interruptores inacessíveis, falta de corrimão nas escadas, degraus inadequados e sem sinalizações ou sem piso antiderrapante, falta de barras de apoio nos banheiros, assentos sanitários de altura inadequada, camas também de alturas inadequadas, cadeiras de altura incorreta e sem apoiadores laterais, obstáculos no caminho, como móveis baixos e fios, presença de animais. ${ }^{3-6,13}$

O presente estudo constatou que as pesquisas brasileiras referentes às quedas em idosos têm dado grande ênfase aos elementos físicos ambientais, como fatores extrínsecos de risco, mas pouco se tem estudado sobre a influência do comportamento e atitude do idoso como contribuição nestes eventos.

Algumas atividades que resultam em quedas se devem a comportamentos imprudentes, a exemplo de subir uma escada dobrável para limpar o topo de um armário. Atitudes de risco por parte dos idosos que nunca caíram e que têm bom estado funcional parecem ser tão importantes quanto a presença ou a exposição ao risco ambiental. Um estudo revelou alta prevalência de comportamentos de risco entre os idosos: entrar na sala quando está escura, não utilizar iluminação noturna e subir em banco para alcançar objetos. ${ }^{14}$ Ribeiro et al. ${ }^{15}$ ressaltam ainda que o uso de calçados inadequados foi relatado em $64,7 \%$ da população idosa utilizada numa pesquisa sobre a influência das quedas na qualidade de vida.

De forma complementar aos comportamentos, as atitudes também são citadas na literatura. Riscos estão presentes em atos rotineiros como sentar ou levantar de camas ou cadeiras; tropeçar em objetos da casa, ou revestimentos do assoalho (tapetes, carpetes e soleiras de portas) e escorregar em superfícies molhadas, ou descendo escadas. Ganança et al. ${ }^{7}$ apontaram as principais tarefas realizadas pelos idosos durante a ocorrência das quedas: deambulação $53,1 \%$; descer/subir escadas $10,9 \%$; transferência postural 9,4\%; e 6,3\% banho. Outro estudo de investigação da história de quedas realizado num hospital terciário revelou resultados semelhantes: deambulação 37,5\%; banho 20\%; levantar da cama $17,5 \%$; caminhar na rua $15 \%$; e outras menos citadas $10 \%{ }^{6}$

A identificação dos fatores de risco significativos causadores de quedas é uma importante etapa no sentido de estabelecer estratégias para prevenção da mesma. Portanto, tendo explicitado estes fatores, faz-se necessário apontar, na literatura pesquisa$\mathrm{da}$, as recomendações sugeridas para intervir na população idosa e no seu ambiente, visando a prevenir tais eventos. As intervenções deverão ajudar os usuários dos serviços de saúde e seus cuidadores, principalmente, a compreender a forma de reduzir a probabilidade de queda, sendo imprescindível que a residência do idoso seja diferenciada, a fim de que ofereça segurança neces- 
sária e minimize o risco das quedas e suas possíveis consequências indesejáveis.

Um ambiente propício e satisfatório para o idoso é aquele que oferece segurança, é funcional, proporciona estímulo e controle pessoal, facilita a interação social, favorece a adaptação às mudanças e é familiar para o idoso. Gianasi et al. ${ }^{10}$ propõem alterações na casa do idoso com vistas a aumentar a atividade física de rotina e facilitar seu deslocamento e equilíbrio. Sugerem ainda eliminar barreiras arquitetônicas, oferecer recomendações de projetos de habitação, divulgando-os para os profissionais e a população associada a idosos. Outros autores também seguiram a mesma linha de intervenção espacial, buscando adequações e modificações no mobiliário tornando-o ideal para idosos. ${ }^{11,13,16}$ As mudanças, sempre que necessárias, devem ser feitas com o consentimento do idoso, pois cada objeto de sua moradia tem um significado afetivo, cuja alteração significa desencadear uma reorganização interna, mas que necessitam ser realizadas para melhorar sua segurança.

A literatura consultada também se refere às intervenções educativas. Numa abordagem mais ampla, Fabrício, Rodrigues e Junior ${ }^{17}$ recomendam aos programas de saúde que estabeleçam protocolos para identificar possíveis riscos causadores de queda. Além disso, durante a avaliação pós-queda deve-se detalhar o acometimento consequente a fatores extrínsecos ou não. Apontam que poderiam ser realizadas visitas domiciliares preventivas, pontuando que a Política
Nacional do Idoso $^{18}$ já aborda este tipo de assistência. Sinalizam que, para isso, estados e municípios teriam que capacitar profissionais de saúde e organizar serviços para que a atenção ao idoso fosse uma política governamental. Perracine \& Ramos ${ }^{5}$ reclamam a utilização de equipe multidisciplinar e a criação de pólos de capacitação profissional para que sejam instaladas intervenções para a adequação arquitetônica dos espaços urbanos e das edificações públicas. Assim como Mathias, Jorge e Andrade (2004), ${ }^{19}$ que também sugerem a diminuição de barreiras arquitetônicas tanto domiciliares quanto públicas, e também a implementação de programas de conscientização dos riscos.

Orientações visando a modificar os comportamentos de risco para garantir movimentos e transferências seguros, sem restringir a possibilidade de vida ativa, bem como instituir ações que previnam lesões sérias, devem ser primordiais. A avaliação periódica da segurança do ambiente doméstico do idoso e referência a cuidados especializados também são requisitos básicos na redução das quedas. Como reforçado por Fabrício, Rodrigues e Junior, ${ }^{17}$ é essencial a conscientização do autocuidado, bem como alertar a família/cuidador para que participem ativamente deste processo.

Pode-se concluir que, na literatura consultada, há conhecimento e soluções em abundância para eliminação dos fatores de risco físicos ambientais relacionados às quedas em idosos. Por sua vez, os elementos 
comportamentais associados a estes eventos contemplados na literatura são ainda pouco estudados, o que pode dificultar tanto a elaboração, como o sucesso das estratégias de prevenção das quedas na população idosa.

Os resultados apresentados até aqui podem servir para estimular novos estudos que contribuam para preencher esta lacuna do conhecimento relacionado à influência dos

\section{REFERÊNCIAS}

1. Pereira SRM, Buksman S, Perracini M, Py L, Barreto KML, Leite VMM. Quedas em idosos. Projeto Diretrizes. Associação Médica Brasileira e Conselho Federal de Medicina. Sociedade Brasileira de Geriatria e Gerontologia [on line] 2001 [acesso em: 2007 mar 31] [9 p]. Disponível em: URL:// http://

www.projetodiretrizes.org.br/ projeto_diretrizes/082.pdf

2. Silva APS, Silva JS. A influência dos fatores extrínsecos nas quedas de idosos. Reabilitar 2003 jul./set; 5(20): 38-42.

3. Gawryszewski VP, Jorge MHPM, Koizumi MS. Mortes e internações por causas externas entre os idosos no Brasil: o desafio de integrar a saúde coletiva e atenção individual. Rev Assoc Med Brás 2004; 50(1): 97-103.

4. Guimarães JMN, Farinatti PTV. Análise descritiva de variáveis teoricamente associadas ao risco de quedas em mulheres idosas. Revista brasileira de medicina do esporte 2005 set./out.; 11(5): 299-305. fatores de risco comportamentais nas quedas em idosos, gerando novas práticas preventivas para atender a esta crescente parcela da população propiciando segurança, independência e uma melhor qualidade de vida.

Apesar da riqueza dos achados, o estudo apresenta como limitação o alcance dos seus resultados, por estarem restritos ao âmbito dos problemas específicos da população idosa brasileira.

5. Perracine MR, Ramos LR. Fatores associados a quedas em uma coorte de idosos residentes na comunidade. Rev Saúde Pública 2002; 36(6): 709-16.

6. Sousa RL, Oliveira SDG, Gonçalves CEF. Investigação da história de queda na clientela idosa de um hospital terciário. JBM; jornal brasileiro de medicina 2004; 87(1): 19-24.

7. Ganança FF, et al. Circunstâncias e consequências de quedas em idosos com vestibulopatia crônica. Rev Bras Otorrinolaringol 2006; 72(3): 388-93.

8. Garcia R, Leme MD, Leme LEG. Evolução de idosos brasileiros com fratura de quadril secundária a quedas. Clinics 2006; 61(6):539-44.

9. Rodrigues KL, Honda CM, Buriti MA. Problema da perda de equilíbrio na terceira idade: fatores de risco e consequências. Revista Fisio\&terapia 2004 fev./mar.; 43(8): 32-7.

10. Gianasi ALA, et al. Recomendação de projeto de habitação para proporcionar qualidade de vida no envelhecimento. Reabilitar 2004; 23(6):51-8. 
11. Ribas VG, Ely VHMB. Parâmetros de projeto para moradia tutelada da terceira idade. Revista Estudos em Design 2002; 5.

12. Tomasini, SLV. Envelhecimento e planejamento do ambiente construído: em busca de um enfoque interdisciplinar. Revista Brasileira de Ciências do Envelhecimento Humano 2005; 2(1): 76-88.

13. Meira EC, et al. Risco de quedas no ambiente físico domiciliar de idosos. Textos Envelhecimento2005; 8(3).

14. Ferrer MLP, Perracine MR, Ramos LR. Prevalência de fatores ambientais associados a quedas em idosos residentes na comunidade em São Paulo, SP. Revista brasileira de fisioterapia 2004; 8(2): 149-54.

15. Ribeiro AP, et al. A influência das quedas na qualidade de vida de idosos. Revista Ciência e Saúde, 0306/ 2006 (Prelo).
16. Barbosa MLJ, Nascimento EFA. Incidência de internações de idosos por motivo de quedas, em um hospital geral de Taubaté. Revista Biociências 2001 jan./ jun; 7(1): 3542.

17. Fabrício SCC, Rodrigues RAP, Costa Junior, ML. Causas e consequências de quedas de idosos atendidos em hospital público. Rev Saúde Pública 2004 fev.; 38(1): 93-9.

18. Mathias TAF, Jorge MHPM, Andrade OG. Morbimortalidade por causas externas na população idosa residente em município da região sul do Brasil. Rev Lat Am Enfermagem 2006 jan./fev;; 14(1):17-24.

19. Política Nacional do Idoso. Lei no 8.842 , de 4 de janeiro de 1994. [acesso em: 10 mar 2007). Disponível em: URL: http://www. cress-se.org.br/pdfs/legislacao_idoso_ 8842.pdf

Recebido: $12 / 2 / 2008$

Revisado; $18 / 4 / 2008$

Aprovado: 4/5/2009 